\title{
Precise Determination of Thickness and Optical Constant of Oxide Layer on Gold by Three-Parameter Ellipsometry
}

\author{
Toshiaki OHTSUKA
}

Received June 3, 1992 ; Accepted July 24, 1992

\begin{abstract}
The oxide layer of gold electrode in 0.005 moldm $^{-3}$ sulphuric acid solution has been investigated by a newly designed three-parameter ellipsometer. The ellipsometer can quantitatively characterize the surface film with the thickness from a submonolayer to a few monolayers from the change of the ellipsometric parameters, $\Psi$ and $\Delta$, and reflectivity, $R$. The oxide layer on gold grows linearly with potential at a thickness-to-potential ratio of $1.2 \mathrm{~nm} \mathrm{~V}^{-1}$. The complex refractive index of the layer simultaneously calculated depends on its thickness, and indicates that the oxide layer is metallic or semiconductive.
\end{abstract}

\section{INTRODUCTION}

The precise determination of thin oxide layer on electrode is of major importance in electrochemistry, metallic corrosion and electrodeposition. The optical measurement including electroreflectance and ellipsometry is one of the promised methods for the characterization of the thin oxide layer.

For the oxide layer on gold electrode during anodic oxidation there have been many works by use of electrochemical and optical techniques 1-5). Usually, the thickness of oxide layer could be estimated under some assumptions. In the case of electrochemical coulometry the density and the composition of the layer have been assumed. The real or imaginary

Department of Applied Chemistry, Nagoya Institute of Technology (Gokiso-cho, Showa-ku, NAGOYA, 466 JAPAN)

Key words ; Ellipsometry, 0xide, Film, Gold, Electrode part of complex refractive index of the layer has been assumed for the estimation by the conventional optical methods. A successful combination of the ellipsometry with the reflectometry was achieved in the study by Cahan et al ${ }^{6,7)}$, where the three optical parameters were simultaneously measured for the formation of the surface layer on noble metals. The thickness and both real and imaginary parts of the complex refractive index can be calculated f'rom the three independent parameters by a. computer simulation with no assumption.

In the present article, a three-parameter ellipsometer has been designed where the reflectivity, $R$, has simultaneously been measured in addition to the ellipsometric parameters, $\Psi$ and $\Delta$. The apparatus described here is sensitive enough to measure a submonolayer fila on electrode. Quantitative characterization for the oxide layer on gold electrode has been performed in the present paper. 


\section{BLLIPSOHBTBR}

The ellipsometer used is schematically show in Fig.1. The main optical components are arranged as the following order; a He-Ne laser with $632.8 \mathrm{~nm}$ mavelength, polarizer (P), quarter-wavelength phase compensator $(C)$, reflection sample (S), rotating analyser (A), and PIN silicon photo-detector. The fluctuation of the laser light intensity is calibrated by monitoring a light beam divided from the main light path by a half mirror. The rotation of the A modulates intensity of polarized reflection light as following.

$I=R\{1+\alpha \cos (2 \omega \mathrm{t})+\beta \sin (2 \omega \mathrm{t})\}$ (1)

where $\omega$ is angular frequency of the rotating analyser and $R$ is an average intensity of reflection light. For the optical P-C-S-A arrangement the Fourier coefficients, $\alpha$ and $\beta$, are related with the relative complex reflection coefficient, $\rho$, of the sample as following ${ }^{8}$ ).

$$
\begin{aligned}
\rho= & \tan \Psi \exp i \Delta \\
= & \frac{1+\alpha}{\beta+i\left(1-\alpha^{2}-\beta^{2}\right) 1 / 2} \\
& \times \frac{\tan C+\rho_{c} \tan (P-C)}{1-\rho_{c} \tan C \tan (P-C)}
\end{aligned}
$$

where $P$ is a polarizer azimuth, $C$ a compensator azimuth, and $\rho_{\mathrm{c}}$ a relative complex transmittance of the compensator. In the apparatus $R \alpha$ and $R \beta$ are measured by the two-phase lock-in amplifier and the average intensity, $R$, by the low-pass filter (a). These values are normalized by the out-put from the low-pass filter (b) to compensate the fluctuation of the laser light intensity. A desk-top computer (NRC PC-9801UV) was used for the accumulation of $\alpha, \beta$, and $R$ as well as the electrochemical data, and for the calculation of $\Psi, \Delta$, and $\Delta R / R$.

So that the high sensitivity was attained, the azimuths of $\mathrm{P}$ and $\mathrm{C}$ were set to make the condition of $\alpha=\beta=0$ for a starting surface free from the oxide. The relative complex reflection coefficient, $\rho_{o}$, can be calculated for the starting surface from equation (3) with $\alpha=\beta=0$.

$$
\begin{aligned}
\rho_{0} & =\tan \Psi_{0} \exp \text { i } \Delta_{0} \\
& =\frac{\tan C+\rho_{\mathbf{c}} \tan (P-C)}{\mathrm{i}\left[1-\rho_{\mathbf{c}} \tan C \tan (P-C)\right]}
\end{aligned}
$$

The values of $\Psi_{0}$ and $\Delta_{0}$ were used for the further calculation of the changes of $\Psi$ and $\Delta$ with the oxide layer formation.

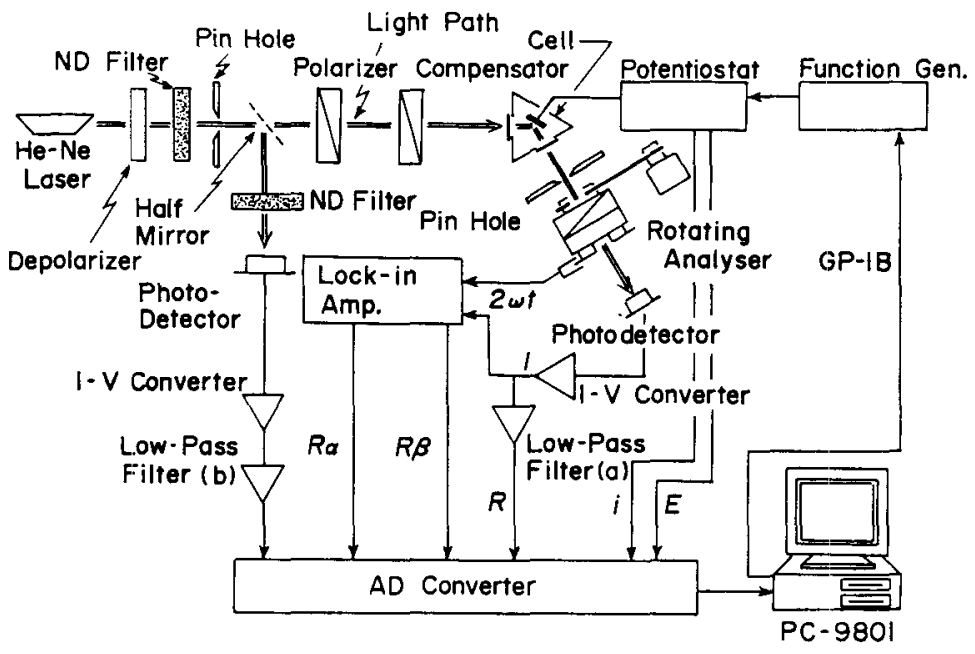

Fig. 1 Scheme of the three-parameter ellipsometer. 
$1+\alpha$

$\tan \Psi \exp$ i $\Delta=$

$$
\begin{array}{r}
\beta+\mathrm{i}\left(1-\alpha^{2}-\beta^{2}\right)^{1 / 2} \\
\mathrm{x} \tan \Psi_{o} \exp \mathrm{i}\left(\Delta_{\mathrm{o}}+\pi / 2\right)
\end{array}
$$

The values of $\alpha, \beta$, and $R$ change with the formation of the oxide layer during potential sweep. From these values and equation (4) the changes in $\Psi$ and $\Delta$ for the film formation were calculated. The $\Psi, \Delta$ and the relative reflectivity change, $\Delta R / R=\left[\left(R-R_{0}\right) / R_{0}\right]$, were next used for the calculation of the complex refractive index and thickness of the oxide layer, where $R_{0}$ corresponds to the reflectivity of the oxide-free surface. The calculation was conducted by the Newton-Rapson successive approximation coded in the Basic program to solve the Fresnel's and Drude's equations for the light reflection on the film-covered surface.

The ellipsometer in the present study has the following technical features.

(1) Real time monitoring; the detection of the Fourier coefficients, $\alpha$ and $\beta$, is made by a phase ${ }^{-}$sensitive analog devise (i.e. the lock-in amplifier) instead of the digital Fourier integration by a computer. The latter technique has usually been used in the conventional rotatinganalyser ellipsometer. The utilization of the lock-in amplifier easily enable the change in $\alpha$ and $\beta$ (or $\Psi$ and $\Delta$ ) to be continuously measured. (2) High sensitivity; the small changes in $\Psi$ and $\Delta$ can be detected by the lock-in amplifier from the optimization of $P$ and $C$ to make the condition of $\alpha=\beta=0$. In the case of the Au electrode in this study the changes of $\Psi$ and $\Delta$ were, respectively, detected with accuracies of $0.01^{\circ}$ and $0.05^{\circ}$ in every $1 \mathrm{~s}$.

\section{EXPERIMRNTAL,}

The electrochemical optical cell in the shape of a triangular prism was made of acrylic acid resin. On two side walls at the cell, the windows of the optically flat glass were fixed for incidence and reflection of light. The angle of incidence for light reflection was $(\pi / 3)$ radian $\left(60.0^{\circ}\right)$. The conventional three-electrode measurement can be made under nitrogen atmosphere in the cell where a working electrode, a platinum plate counter electrode, and a Luggin capillary connected to a reference electrode are inserted from the top cover of the cell.

The gold electrode was a polycrystal sheet $1 \mathrm{~mm}$ thick cut to $10 \times 10 \mathrm{~mm}^{2}$ with a small tab for electric connection. The electrode was mechanically polished with alumina abrasives through to $0.05 \mu \mathrm{m}$ diameter, degreased in acetone by an ultrasonic washer, and then immersed in the $1: 1$ mixture of concentrated sulphuric and nitric acids. Before experimental setup the electrode was rinsed by deionized-distilled water.

The potential was measured by an $\mathrm{Ag}-\mathrm{AgCl}$ reference electrode in saturated KCl solution, and the experiment was made

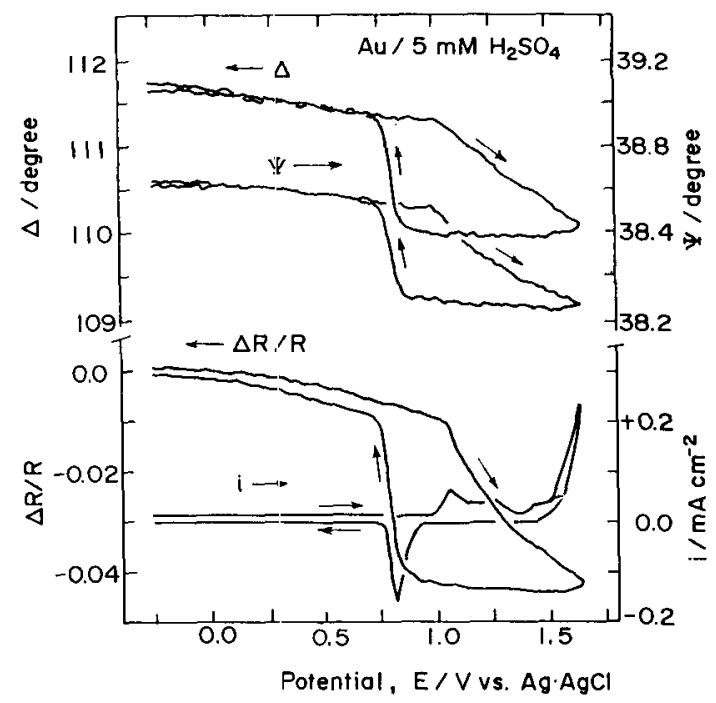

Fig. 2 Change of ellispometric parameters $(\Psi$ and $\Delta)$, reflectivity $(R)$, and current density ( $i)$ during potential sweep at $0.01 \mathrm{Vs}^{-1}$ of gold electrode in $0.005 \mathrm{~mol} \mathrm{\textrm {dm } ^ { - 3 }}$ sulpuric acid solution. 
at room temperature about $26^{\circ} \mathrm{C}$. The electrolyte of $\mathrm{H}_{2} \mathrm{SO}_{4}$ prepared from the analytical grade reagent and deionizeddistilled water was deoxygenated by bubbling pure nitrogen for $12 \mathrm{~h}$ before use.

\section{RESULTS}

The anodic polarization was made by potential sweep at a sweep rate of 0.01 $V s^{-1}$ from $-0.26 \mathrm{~V}$ to $1.64 \mathrm{~V}$ in $0.005 \mathrm{~mol}$ $\mathrm{dm}^{-3}$ sulphuric acid solution. Figure 2 shows the result in which $\Psi$ in the relative amplitude attenuation $(\tan \Psi)$, $\Delta$ of the relative phase retardation, $\Delta R / R$ of the relative reflectivity change, and current density, $i$, are plotted against the potential, $E$. The $\Delta R / R$ refers to the reflectivity at $0.00 \mathrm{~V}$. In the electric double layer region up to $1.00 \mathrm{~V}$ in the forward direction of the sweep and below $0.75 \mathrm{~V}$ in the backward direction, the deviation of $\Psi, \Delta$ and $\Delta R / R$ is small. The large optical change at the forward sweep is seen at potential more positive than $1.00 \mathrm{~V}$. The formation of oxide layer takes place in this potential region. After the reverse of potential at $1.64 \mathrm{~V}$ the recovery of $\Psi, \Delta$ and $\Delta R / R$ is small. The reduction of the oxide layer occurs at a potential region from 0.90 to $0.76 \mathrm{~V}$ where a cathodic current peak is observed.

\section{DISCOSSION}

\section{1. ELBCTRIC DOUBLE LAYBR RBGION}

The small changes of $\psi, \Delta$ and $\Delta R / R$ were observed in the electric double layer region. Three possible models have been proposed on the origin of the small changes. (1) Change of the optical constant of gold electrode which is caused by a change in the free electron concentration at the surface region of gold with potential 9,10$)$. (2) Change of the optical constant of the electric double layer in the neighbor of the gold electrode surface which is caused by changes in ionic concentration and structure including the water dipole reorientation ${ }^{11}$. (3) Change in the dielectric property of the transient layer from the gold electrode to the electric double layer, the optical property of which continuously varies in the direction normal to the surface ${ }^{12}$ ).

Figure 3 shows a result of the variation of the complex refractive index of the gold electrode calculated from the first model. In Fig. 3 the reflectance was theoretically calculated from the $\mathrm{n}$ and $\mathbf{k}$, which should be compared with the $\Delta R / R$ in Fig. 2. The $n-i k$ of the gold is seen to change from $0.7356-\mathrm{i} 2.640$ at $0.00 \mathrm{~V}$ to $0.7453-\mathrm{i} 2.618$ at $+1.00 \mathrm{~V}$. The relative reflectivity change, $\Delta R / R$, from $0.00 \mathrm{~V}$ to $1.00 \mathrm{~V}$ is theoretically calculated to be $8.2 \times 10^{-3}$, as shown in Fig. 3. The experimental result in Fig. 2 shows $\Delta R / R=$ $9.3 \times 10^{-3}$. The difference between them is

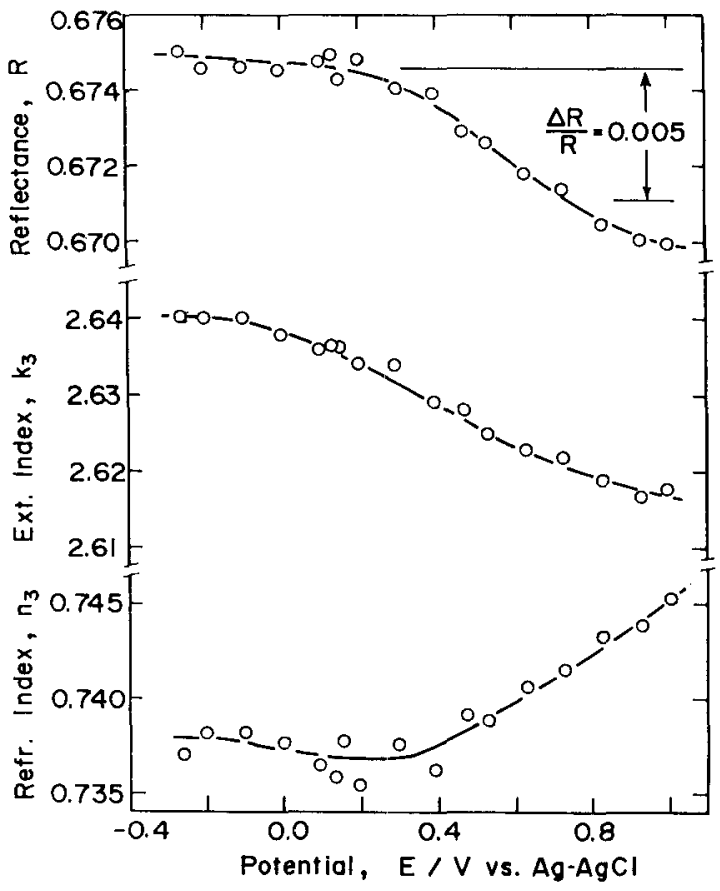

Pig. 3 Change of complex refractive index (n-ik) of gold electrode with potential in the electric double layer potential region. The reflectance was theoretically calculated from the $(n-i k)$ value. 
sma1l and accordingly it may be suggested that the main contribution to the changes of $\Psi, \Delta$, and $\Delta R / R$ is the change of the optical property at the gold electrode surface. The small difference may indicate that a small contribution of the electric double layer at the electrolyte side is included.

In the second and third models an effective interfacial layer has been assumed, the optical property of which is different from thase of the electrode substrate and the electrolyte. The average thickness and complex refractive index of the effective layer can be estimated from the deviation of $\Psi, \Delta$ and $\Delta R / R$ from the values at $0.00 \mathrm{~V}$ by an assumption of the simple homogeneous layer. The calculation indicated, for example, that the average thickness and complex refractive index of the effective layer were, respectively, $0.30 \mathrm{~nm}$ and 1.9 -i1.2 at $1.00 \mathrm{~V}$. The thickness is reasonable for the effective layer. The complex refractive index calculated may be too large for the interfacial layer of the electrolyte side, and accordingly the second model should be excluded for the explanation. For the third model, since the distribution of the dielectric property in the transient layer has not been found exactly, the validity of the model could not be judged from the experimental results.

The complex refractive index of the gold substrate must be initially fed for the calculation of the optical constant and thickness of the oxide layer in the following section,. The index estimated from the first model at $1.00 \mathrm{~V}$ just below the oxide layer formation is utilized for the calculation.

\section{2.0xide Layer}

Figure 4 shows the complex refractive index, $n_{\mathbf{z}}-i k_{\mathbf{z}}$, and thickness, $d$, of the oxide layer in the high potential region. The calculation was made from the changes of $\Psi, \Delta$ and $\Delta R / R$ from the values at

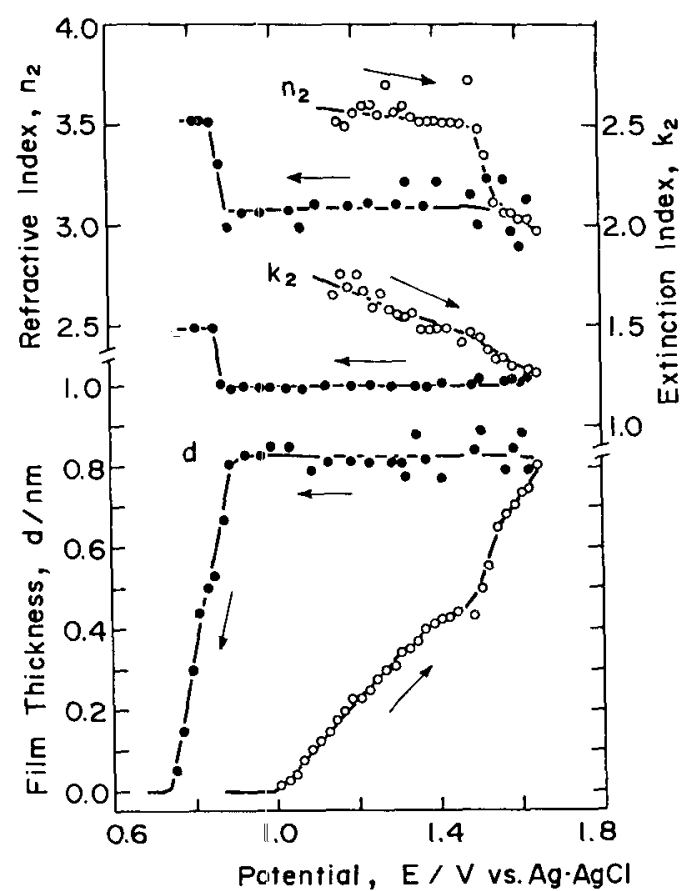

Fig. 4 Complex refractive index and thickness of oxide layer on gold electrode in $0.005 \quad \mathrm{~mol} \mathrm{dm}^{-3}$ sulpfuric acid solution. The complex refractive index of the gold substrate is $0.7453-\mathrm{i} 2.618$ which was calculated from the values of $\Psi$ and $\Delta$ at $1.00 \mathrm{~V}$.

$1.00 \mathrm{~V}$. In potentials between 1.00 and $1.15 \mathrm{~V}$ at the forward sweep and between 0.70 and $0.65 \mathrm{~V}$ at the backward sweep the changes of $\Psi, \Delta$ and $\Delta R / R$ were so small that the three quantities of $\mathbf{n}_{2}$, $\mathbf{k}_{2}$, and $d$ might not be determined precisely. A complex refractive index of 3.66-il.66 was assumed in the potential regions. The index corresponds to an average value estimated at potentials between 1.15 to $1.25 \mathrm{~V}$ at the forward sweep.

The three quantities of $\mathbf{n}_{\mathbf{2}}, \mathrm{k}_{\mathbf{z}}$, and $d$ were estimated from the Newton-Rapson successive approximation in the potentials more positive than $1.15 \mathrm{~V}$ at the forward sweep or than $0.70 \mathrm{~V}$ at the backward sweep. The approximation continued until 
the difference between the experimental and calculated values was reduced below the predetermined limits of \pm 0.0001 for $\tan \Psi, \quad \pm 0.0005$ for $\cos \Delta$ and \pm 0.0001 for $\Delta R / R$. The values of $\mathrm{n}_{2}, \mathrm{k}_{\mathbf{2}}$, and $d$ estimated were scattered due to the fluctuation of measurement values in $0.01^{\circ}$ for $\Psi, 0.05^{\circ}$ for $\Delta$, and 0.001 for $\Delta R / R$. The scattering ranges depended on the amounts of changes of $\Psi, \Delta$, and $\Delta R / R$. Typically the scattering ranges in the potential region from 1.60 to $1.00 \mathrm{~V}$ during the backward sweep are about \pm 0.12 for $\mathrm{n}_{2}, \pm 0.05$ for $k_{2}$ and $\pm 0.05 \mathrm{~nm}$ for $d$.

As seen in Fig. 4, the thickness increases with the increase of potential at a rate of $1.2 \mathrm{~nm} \mathrm{~V}^{-1}$. The refractive index, $\mathrm{n}_{\mathbf{2}}$, changes with the layer growth. The index is seen to be a relatively high value of about 3.5 in potential less positive than $1.5 \mathrm{~V}$ during the forward sweep, and then decreases to $\mathbf{n}_{\mathbf{z}}=3.1$ at $1.64 \mathrm{~V}$. The extinction index, $\mathbf{k}_{2}$, also decreases with the increase of potential from $k_{\mathbf{z}}=1.7$ at $1.2 \mathrm{~V}$ to 1.25 at $1.64 \mathrm{~V}$. During the backward potential sweep from $1.64 \mathrm{~V}$, the thickness is kept constant at $0.86 \mathrm{~nm}$. In the potential region of the constant film thickness the refractive and extinction indices are almost constant within the error limits. The reduction of oxide layer takes place between potentials of $0.90 \mathrm{~V}$ and of $0.76 \mathrm{~V}$. $0 \mathrm{n}$ the voltammogram in Fig. 2, the cathodic current peak is observed at the potential region. The cathodic reduction of the oxide layer is found to require a considerable large overvoltage, which agrees with the results in the literature $5-8,13,15,16)$. After the reduction the values of $\Psi$ and $\Delta$ return to the values of the starting surface at the forward sweep, as shown in Fig. 2, while the $\Delta R / R$ is a slightly smaller value than that of the starting surface. The difference in $\triangle R / R$ may be explained from a change of surface morphology which is caused by the cycle of the oxide layer formation and removal.

The complex refractive index calculated is dependent on its thickness in potentials less positive than $1.5 \mathrm{~V}$ at the forward sweep. The layer in this potential region is considered to be an chemisorbed one rather than a phase oxide. It is conceivable that the index is much larger than that of the $0^{2-} / \mathrm{OH}^{-}$chemisorbed layer. The index may thus correspond to that of the effective layer including the chemisorbed $0^{2-} / \mathrm{OH}^{-} \quad$ layer and the charge-transferred surface of the gold.

In the potential more positive than 1.5 $V$ at the forward sweep, the layer may consist of a phase oxide. In the earlier electrochemical and photoelectrochemical studies, the composition of the phase oxide is assumed to be $\mathrm{Au}_{2} \mathrm{O}_{3}{ }^{15,16)}$, and our optical measurement however provides no information on the composition.

The complex refractive index obtained here is comparable with those reported by Bolb et al 4) and by Horkans et al ${ }^{7}$, The former authors measured the two reflectivity changes of $\mathrm{p}^{-}$and s-polarization with the film formation on gold electrode at $1.6 \mathrm{~V}$ vs. RHE in $1 \mathrm{~mol}$ $\mathrm{dm}^{-3}$ perchloric acid solution. They calculated the complex dielectric constant at various wavelengths with a film thickness of $0.6 \mathrm{~nm}$ which was estimated from the coulometry. From their complex dielectric constant at 633 nm wavelength the complex refractive index becomes 2.95-i1.56 which is not much different from the value described here. The latter authors measured the oxide layer in $1 \mathrm{~mol}$ $\mathrm{dm}^{-3}$ perchloric acid solution by the three-parameter ellipsometry and reported the complex refractive index of $3.3-\mathrm{i} 1.3$ and the thickness of $0.54 \mathrm{~nm}$ in a wavelength region from 602 to $715 \mathrm{~nm}$ at a potential of $1.65 \mathrm{~V}$ vs. NHE (corresponding to $1.45 \mathrm{~V}$ vs. $\mathrm{Ag}-\mathrm{AgCl}$ ). These values are in agreement with the 
present ones at the same potential.

From the value of complex refractive index, specially from the large $k$ value, it may be suggested that the thin oxide layer initially formed on gold is of a metallic or semiconductive character.

\section{CONCLUSION}

(1) A newly designed three-parameter ellipsometer is sensitive enough to characterize a submonolayer on electrode.

(2) The oxide layer on gold electrode grows 1 inearly with potential at a rate of $1.2 \mathrm{~nm} \mathrm{~V}^{-1}$ in $0.005 \mathrm{~mol} \mathrm{dm}^{-3}$ sulphuric acid solution.

(3) The complex refractive index of the oxide layer from a submonolayer to a few monolayers indicates that the layer has a metallic or semiconductive character.

I acknowledge the financial support of the Shimazdu Science Foundation.

\section{REFERBNCBS}

1) W. E. Reid and J.Kruger, Nature, 203, 402 (1964)

2) D.F.A. Koch and D.E. Sca ife, J.Electrochem. Soc., 113,302(1966)
3) R.S. Sirohi and M.A. Genshow, J.Electrochem. Soc., 116,1910(1969)

4) D. M. Kolb and J.D.E.McIntyre, Surf. Sci. , 28, 321(1971)

5) J.P. Hoare, J.Electrochem. Soc., 131, 1808(1984)

6) B. D. Cahan, J.Horkans and E. Yeager, Surf. Sci., 37, 559(1973)

7) J. Horkans, B. D. Cahan and B. Yeager, ibid. , 46, 1(1974)

8) R. M. A. Azzam and N.M. Bashara, "Ellipsometry and polarized light", North Holland, Amesterdam (1977)

9) A. Prostak and W. N. Hansen, Phys. Review, $160,600(1967)$

10)W. N. Hansen, Surf. Sci., 16, 205(1969)

11)J. Feinleib, Phys. Rev. Letters, 16, 1200(1966)

12)W. J. Plieth, J. de Phys. Colloq., C5, 215(1977)

13)M. Seo, X.C. Jian and N. Sato, J. Electrochem. Soc., 134,3094(1987)

14) R. - F. Lin and T.R. Beck, J. Electrochem. Soc. ibid., 123, 1145 (1976)

15)T. Watanabe and H. Gerischer, J.Electroanal. Chem., 117, 185(1981)

16)T. Watanabe and H. Gerisher, J.Electroanal. Chem., 122, 73(1981) 